\title{
The relationship between changes in vitamin A, vitamin $E$, and oxidative stress levels, and pregnancy outcomes in patients with gestational diabetes mellitus
}

\author{
Huashu Ma, Zongxu Qiao, Na Li, Yefang Zhao, Shasha Zhang \\ Department of Obstetrics, Xingtai People's Hospital Affiliated to Hebei Medical University, Xingtai, China \\ Contributions: (I) Conception and design: H Ma, Z Qiao; (II) Administrative support: N Li; (III) Provision of study materials and patients: H Ma, Z \\ Qiao, Y Zhao, S Zhang; (IV) Collection and assembly of data: All authors; (V) Data analysis and interpretation: H Ma, N Li, Y Zhao, S Zhang; (VI) \\ Manuscript writing: All authors; (VII) Final approval of manuscript: All authors. \\ Correspondence to: Huashu Ma. 13-2-902, North District, Xingtai 054000, China. Email: mahuashu@126.com.
}

Background: Gestational diabetes mellitus (GDM) is one of the most common complications during pregnancy. This study aims to analyze the relationship between the changes in vitamin A, vitamin E, and oxidative stress levels, and pregnancy outcomes in GDM patients.

Methods: A total of 104 GDM patients admitted to our hospital between January 2018 and January 2019 were selected as the study group, and 126 pregnant women who received healthy examinations in the same hospital during the same period were selected as the control group. Vitamin A, E, and oxidative stress [total antioxidant capacity (TAOC), malondialdehyde (MDA), red blood cell glutathione (GSH), and superoxide dismutase (SOD)] between the two groups were compared. Age, pre-pregnancy body mass index (BMI), prepregnancy waist-to-hip ratio (waist/hip circumference), parity, gravidity, glycosylated hemoglobin, and other related index levels were collected for the GDM patients. Multivariate logistic regression analysis was used to analyze the risk factors affecting the pregnancy outcomes of the GDM patients.

Results: The levels of vitamin A, SOD, GSH, and TAOC in the study group were significantly lower than those in the control group, and the levels of vitamin $\mathrm{E}$ and MDA were significantly higher than those in the control group $(\mathrm{P}<0.05)$. Of the 104 GDM patients, 43 had adverse pregnancy outcomes, and 61 had no adverse pregnancy outcomes. There was a significant difference between the adverse and non-adverse pregnancy outcome groups in the levels of glycosylated hemoglobin, vitamin A, vitamin E, MDA, SOD, GSH, and TAOC $(\mathrm{P}<0.05)$. Analysis of the unconditional multivariate logistic regression model showed that the expression levels of glycosylated hemoglobin, vitamin A, vitamin E, MDA, SOD, GSH, and TAOC were independent risk factors affecting the pregnancy outcomes of GDM patients $(\mathrm{P}<0.05)$.

Conclusions: Vitamin A, E, and oxidative stress levels are abnormally expressed in GDM, and are independent risk factors affecting the pregnancy outcomes in GDM patients. Therefore, the clinical monitoring of oxidative stress levels and vitamin supplementation should be increased, as these may be of great significance in improving pregnancy outcomes in GDM patients.

Keywords: Gestational diabetes mellitus (GDM); vitamin A; vitamin E; oxidative stress; pregnancy outcomes

Submitted Apr 11, 2021. Accepted for publication May 26, 2021.

doi: 10.21037/apm-21-1036

View this article at: http://dx.doi.org/10.21037/apm-21-1036 


\section{Introduction}

Gestational diabetes mellitus (GDM) is defined as the onset of diabetes occurring during pregnancy due to abnormal maternal glucose metabolism and is a common complication of pregnancy. Relevant research shows that GDM seriously endangers the health of mothers and babies and has an incidence of $1-5 \%$, which appears to be increasing yearly $(1,2)$. Therefore, it is of utmost importance to efficiently diagnose and treat GDM to improve the prognosis of patients with this condition.

A large number of studies have shown that vitamins A and $\mathrm{E}$ are essential fat-soluble micronutrients that promote human metabolic processes and play an important role in the formation of DNA and RNA in maintaining the stability and integrity of mucosal tissues (3). Another study reports that vitamin $\mathrm{A}$ is an important cofactor of extracellular matrix proteins, and vitamin $\mathrm{E}$ is an important anti-free radical oxidation substance in tissues, and both of these vitamins have significant roles in maintaining the growth and development of the body and maintaining normal physiological functions (4). In addition, previous studies have found that there is an imbalance of oxidative stress in GDM patients, which can cause damage to vascular and pancreatic $\beta$-cells and affect pregnancy outcomes (5). In this study, we investigated the relationship between changes in vitamin $\mathrm{A}$, vitamin $\mathrm{E}$, and oxidative stress levels, and pregnancy outcomes in GDM patients. We present the following article in accordance with the STROBE reporting checklist (available at http://dx.doi.org/10.21037/apm-211036).

\section{Methods}

\section{General information}

A total of 104 patients with GDM admitted to our hospital between January 2018 and January 2019 were selected as the study group and were aged 22-34 years (mean age $27.03 \pm 3.85$ years). The inclusion criteria were as follows: (I) patients who met the diagnostic criteria for GDM after glucose tolerance test screening; (II) the gestational stage of the patient was 24-28 weeks; (III) patients with singleton pregnancies; (IV) patients with complete clinical data. The exclusion criteria were as follows: (I) a family history of diabetes; (II) a diagnosis of diabetes before pregnancy or during pregnancy; (III) patients who had taken glucose or lipid metabolism drugs in the previous six months; (IV) patients with mental or communication disorders.

In addition, 126 pregnant women who received health examinations in the same hospital during the same period and had healthy results were enrolled in the control group, with a mean age of $27.25 \pm 3.86$ years. There was no significant difference in the general data of the two groups $(\mathrm{P}>0.05)$. This study was approved by the Research Ethics Committee of Xingtai People's Hospital: (2019) 064. All procedures performed in this study involving human participants were in accordance with the Declaration of Helsinki (as revised in 2013). The informed consent was taken from all the patients.

\section{Detection methods}

The morning after admission, $3 \mathrm{~mL}$ of fasting venous blood was drawn from the patients and collected in a lithium heparin anticoagulation tube. After being centrifuged at $3,000 \mathrm{rpm} \times 15 \mathrm{~min}$ at $4{ }^{\circ} \mathrm{C}, 400 \mu \mathrm{L}$ of plasma from each sample was sent for further testing. A high-performance liquid chromatography (HPLC) analyzer (Agi-lent 1260, USA) was used to quantitatively determine the concentration of vitamin $\mathrm{A}$ and vitamin $\mathrm{E}$. An automatic biochemical analyzer was used to detect the total antioxidant capacity (TAOC) with the $\mathrm{Fe}^{3+}$ reduction method, the level of malondialdehyde (MDA) was detected with the thiobarbituric acid reactant colorimetric method, red blood cell glutathione (GSH) was detected with the dithionitrobenzoic acid colorimetric method, and the level of superoxide dismutase (SOD) was detected with pyrogallol auto-oxidation inhibition colorimetry. The procedural steps were carried out in strict accordance with the instrument's manual. The normal reference range of vitamin $\mathrm{A}$ is $0.3-$ $0.7 \mathrm{mg} / \mathrm{L}$ (6). A level of vitamin A less than $0.3 \mathrm{mg} / \mathrm{L}$ indicates a deficiency, whereas a level over $0.7 \mathrm{mg} / \mathrm{L}$ indicates an excess of vitamin $\mathrm{A}$. The normal reference range of vitamin $\mathrm{E}$ is $5.0-20.0 \mathrm{mg} / \mathrm{L} \mathrm{(6).} \mathrm{Vitamin} \mathrm{E}$ deficiency is defined as a level less than $5.0 \mathrm{mg} / \mathrm{L}$, and a vitamin $\mathrm{E}$ excess is defined as a level over $20 \mathrm{mg} / \mathrm{L}$.

\section{Follow-up methods}

All subjects in both groups underwent routine obstetric examinations to closely monitor their health and that of their fetuses, and the final pregnancy outcomes of the two groups were recorded. Diagnostic criteria for adverse pregnancy outcomes were as follows: during follow-up, the patient required hospital admission for treatment of 
Table 1 Comparison of vitamin A, vitamin E, and oxidative stress levels in pregnant women between the two groups $\left(\bar{x}_{ \pm} \pm \mathrm{s}\right)$

\begin{tabular}{|c|c|c|c|c|c|c|c|}
\hline Group & $\mathrm{n}$ & Vitamin A (mg/L) & Vitamin E (mg/L) & MDA (nmol/mL) & $\mathrm{SOD}(\mathrm{U} / \mathrm{L})$ & $\mathrm{GSH}(\mathrm{mg} / \mathrm{gHB})$ & TAOC $(\mathrm{U} / \mathrm{mL})$ \\
\hline Study group & 104 & $0.15 \pm 0.08$ & $24.33 \pm 2.81$ & $23.84 \pm 2.06$ & $6.19 \pm 2.51$ & $59.71 \pm 5.42$ & $9.33 \pm 1.58$ \\
\hline Control group & 126 & $0.57 \pm 0.09$ & $8.41 \pm 2.06$ & $12.75 \pm 1.95$ & $22.06 \pm 2.80$ & $79.42 \pm 5.61$ & $12.51 \pm 2.34$ \\
\hline$P$ & - & $<0.001$ & $<0.001$ & $<0.001$ & $<0.001$ & $<0.001$ & $<0.001$ \\
\hline
\end{tabular}

MDA, malondialdehyde; SOD, superoxide dismutase; GSH, glutathione; TAOC, total antioxidant capacity.

complications, such as fetal macrosomia (greater than normal birth weight), neonatal hypoglycemia, premature rupture of membranes, placental abruption, premature delivery, fetal distress, threatened abortion, preeclampsia, threatened premature delivery, too much or too little amniotic fluid, fetal growth restriction, stillbirth, or severe fetal malformations.

\section{Statistical analysis}

All data in this study were statistically analyzed using SPSS 22.0 software (IBM, New York, USA). The measurement data are described as the mean \pm standard deviation $(\bar{x} \pm s)$ and analyzed by $t$-tests. The count data are expressed as the pass rate or composition ratio and analyzed by the chisquared $\left(\chi^{2}\right)$ test. Multivariate logistic regression was used to analyze the risk factors affecting the pregnancy outcomes of GDM patients. Results with $\mathrm{P}<0.05$ were considered to be statistically significant.

\section{Results}

Comparison of the levels of vitamin $A$, vitamin $E$, and oxidative stress between the two groups of pregnant women

The levels of vitamin A, SOD, GSH, and TAOC in the study group were significantly lower than those in the control group $(\mathrm{P}<0.05)$, and the levels of vitamin $\mathrm{E}$ and MDA were significantly higher than those in the control group $(\mathrm{P}<0.05$, Table 1).

\section{Pregnancy outcomes of 104 cases with GDM}

Among the total 104 cases of GDM, 41 cases experienced adverse pregnancy outcomes and the remaining 61 cases had no adverse pregnancy outcomes. According to their pregnancy outcomes, the patients were divided into an adverse pregnancy outcome group $(n=43)$ and a non-adverse pregnancy outcome group $(\mathrm{n}=61)$.

\section{Univariate analysis of pregnancy outcome in patients with GDM}

The results of the univariate analysis showed that there was no significant difference in age, pre-pregnancy body mass index (BMI), pre-pregnancy waist-to-hip ratio, gravidity, and parity between the adverse and non-adverse pregnancy outcome groups $(\mathrm{P}>0.05)$. There were statistically significant differences in the levels of glycosylated hemoglobin, vitamin A, vitamin E, MDA, SOD, GSH, and TAOC between the adverse and non-adverse pregnancy outcome groups $(\mathrm{P}<0.05$, Table 2).

\section{Significance and assignment of factors to be analyzed that affect the pregnancy outcome of GDM patients}

The significance and assignment of the factors to be analyzed are shown in Table 3.

\section{Multivariate analysis of factors affecting pregnancy outcomes in GDM patients}

According to the analysis of the unconditional multivariate logistic regression model, results showed that the expression levels of glycosylated hemoglobin, vitamin A, vitamin E, MDA, SOD, GSH, and TAOC were independent risk factors that affected the pregnancy outcomes of GDM patients $(\mathrm{P}<0.05$, Table 4).

\section{Discussion}

GDM is a common disease that causes metabolic dysfunction in pregnant women. With the improvement in people's living standards, the incidence of GDM is increasing yearly. Hyperglycemia during pregnancy endangers maternal health and has adverse effects on the fetus (7). A large number of studies at home and abroad indicate that exploring the risk factors affecting the pregnancy outcomes of GDM patients and providing 
Table 2 Univariate analysis of pregnancy outcomes in patients with GDM

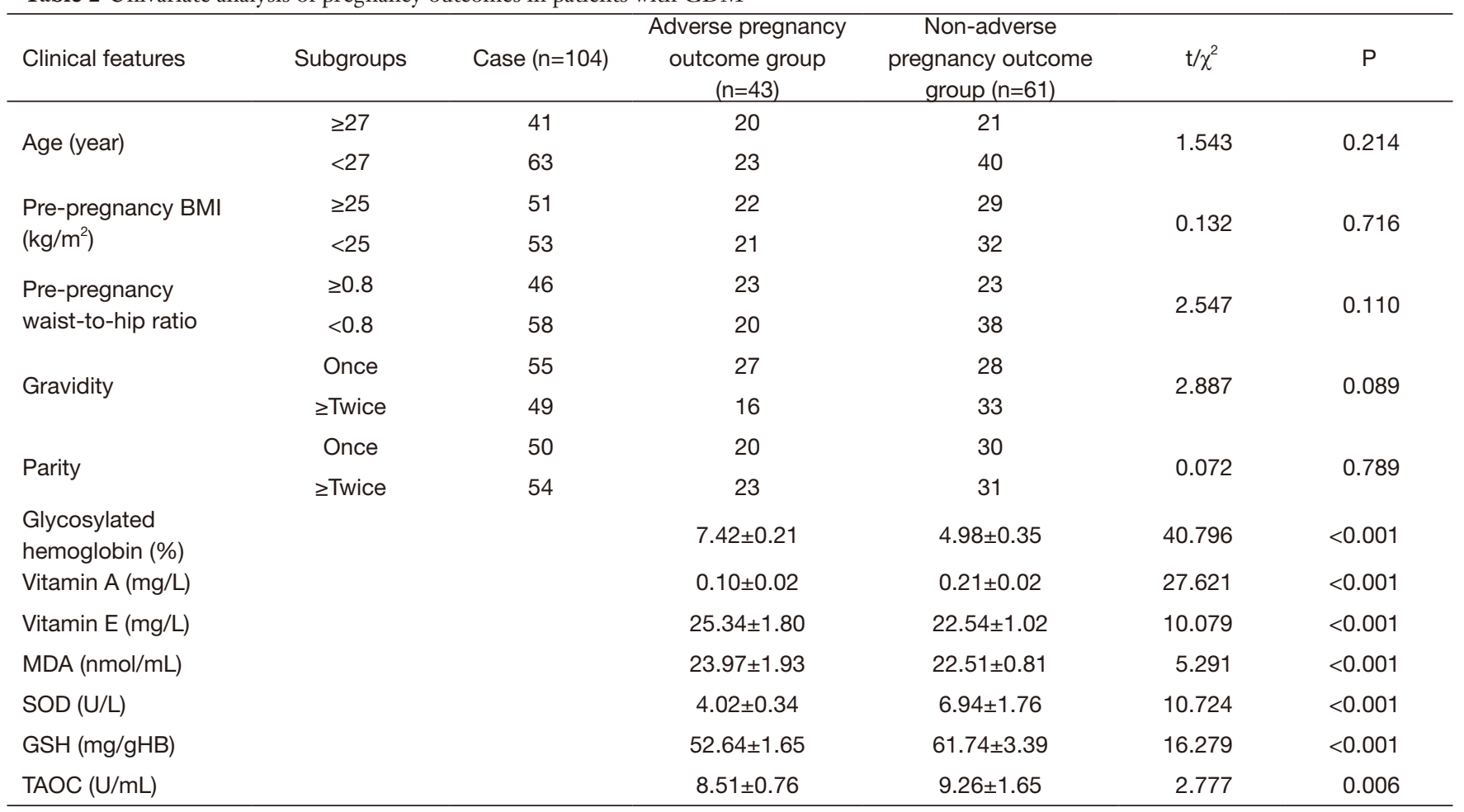

GDM, gestational diabetes mellitus; BMI, body mass index; MDA, malondialdehyde; SOD, superoxide dismutase; GSH, glutathione; TAOC, total antioxidant capacity.

Table 3 Significance and assignment of factors to be analyzed

\begin{tabular}{lcc}
\hline Factor & Significance & Assignment \\
\hline$X_{1}$ & Glycosylated hemoglobin & $0=$ normal; $1=$ abnormal \\
$X_{2}$ & Vitamin A & $0=$ normal; $1=$ lower \\
$X_{3}$ & Vitamin E & $0=$ normal; $1=$ lower \\
$X_{4}$ & MDA & $0=$ normal; $1=$ lower \\
$X_{5}$ & SOD & $0=$ normal; $1=$ lower \\
$X_{6}$ & GSH & $0=$ normal; $1=$ lower \\
$X_{7}$ & TAOC & $0=$ normal; $1=$ lower \\
\hline
\end{tabular}

MDA, malondialdehyde; SOD, superoxide dismutase; GSH, glutathione; TAOC, total antioxidant capacity.

targeted intervention measures have an important clinical value for improving the pregnancy outcomes of GDM patients (8).

In recent years, the scientific community has gained a deeper understanding of the important role that vitamins, such as vitamin A and vitamin E, play in a variety of life activities. During pregnancy, vitamin A has a major impact on the maintenance of cell division, fetal organs, skeletal development, vision, and the immune system. Once vitamin
A is lacking, it will not only affect the normal growth and development of the embryo but may also cause congenital diseases, including pulmonary hypoplasia, central nervous system malformations, and bone deformity of the fetus $(9,10)$. Relevant studies using animal models have shown that vitamin A deficiency leads to the abnormal synthesis of cellular glycoprotein intermediates, accelerates cell division, and induces cell apoptosis, as well as inducing inflammation in the body (11). Vitamin E is an important 
Table 4 Multivariate analysis of pregnancy outcomes in patients with GDM

\begin{tabular}{|c|c|c|c|c|c|}
\hline Factor & Regression coefficient & SEM & Wald $\chi^{2}$ & $P$ & OR $(95 \% \mathrm{Cl})$ \\
\hline $\mathrm{X}_{1}(0$ vs. 1$)$ & 0.915 & 0.205 & 7.459 & 0.025 & $2.49(1.670-3.731)$ \\
\hline $\mathrm{X}_{2}(0$ vs. 1$)$ & 0.932 & 0.151 & 9.184 & $<0.001$ & $2.540(1.889-3.414)$ \\
\hline $\mathrm{X}_{4}(0$ vs. 1$)$ & 0.946 & 0.152 & 8.595 & 0.011 & 2.57 (1.911-3.469) \\
\hline $\mathrm{X}_{5}(0$ vs. 1$)$ & 1.056 & 0.423 & 10.480 & $<0.001$ & $2.875(1.255-6.587)$ \\
\hline $\mathrm{X}_{7}(0$ vs. 1$)$ & 0.844 & 0.156 & 10.274 & $<0.001$ & $2.326(1.713-3.157)$ \\
\hline
\end{tabular}

GDM, gestational diabetes mellitus; SEM, standard error of the mean; OR, odds ratio; $\mathrm{Cl}$, confidence interval.

fat-soluble antioxidant on the cell membrane and has many functions, such as maintaining fertility, preventing cardiovascular disease, improving immunity, and has antitumor, anti-aging, and anti-oxidation properties (12). On the one hand, vitamin $\mathrm{E}$ can protect cell membranes and organelle membranes from oxidative damage by blocking the free radical chain reaction and maintains the integrity and stability of cells and organelles to ensure normal cell functioning; on the other hand, it can also regulate leucoregulin, prostaglandin, coagulin, and the synthesis of cytokines $(13,14)$. A recent study reported that the level of oxidative stress in patients with GDM was significantly increased and that the peroxidation state damaged pancreatic $\beta$-cell functioning and aggravated diabetes (15). MDA is the end product of free radical oxidized lipids, which can reflect the severity of oxidative damage. GSH, SOD, and TAOC are all effective indicators reflecting antioxidant capacity and play an important role in the elimination of internal oxygen free radicals, the promotion of the free radical defense system composition, and antioxidant capacity (16). The results of our study showed that the levels of vitamin A, SOD, GSH, and TAOC of GDM patients were significantly lower than those of healthy pregnant women, and the levels of vitamin $\mathrm{E}$ and MDA were significantly higher than those of healthy pregnant women, indicating that vitamin A, vitamin $\mathrm{E}$, and oxidative stress levels play an important role in patients with GDM.

In addition, our follow-up results showed that 43 of the 104 GDM patients had adverse pregnancy outcomes. GDM can cause vascular disease and abnormal energy metabolism, which enables the fetus to absorb too much sugar, resulting in larger than average babies, an increased risk of delivery, and ultimately a series of adverse outcomes $(17,18)$. To clarify the risk factors affecting the pregnancy outcomes of GDM patients, this study tested the levels of glycosylated hemoglobin, vitamin A, vitamin E, MDA, SOD, GSH, and TAOC in the two groups and found that the expression levels of glycosylated hemoglobin, vitamin A, vitamin E, MDA, SOD, GSH, and TAOC were independent risk factors affecting the pregnancy outcomes of GDM patients $(\mathrm{P}<0.05)$. The mechanism may be that the decrease in vitamin $\mathrm{A}$ and the increase of vitamin $\mathrm{E}$ could promote inflammation, increase oxidative stress, dilate blood vessel functioning, and aggravate placental endothelial damage. Additionally, the results of this study also showed that the oxidative stress level of GDM patients was increased, indicating that antioxidant capacity was decreased. An unsustainably high glucose status leads to glycosylation of antioxidant enzymes, a decrease in the activities of GSH, SOD, and TAOC, and dysfunction of pancreatic $\beta$-cells, affecting the regulation of blood glucose and increasing the risk of adverse pregnancy outcomes (19-21).

In summary, vitamin $\mathrm{A}$, vitamin $\mathrm{E}$, and oxidative stress levels are abnormally expressed in patients with gestational diabetes and are all independent risk factors affecting pregnancy outcomes in patients with GDM. Therefore, in clinical settings, the monitoring of oxidative stress and vitamin supplementation should be increased, as these may be of great significance in improving pregnancy outcomes in GDM patients.

\section{Acknowledgments}

Funding: This study was approved by the 2019 Hebei Province Medical Science Research Project (20191680).

\section{Footnote}

Reporting Checklist: The authors have completed the STROBE reporting checklist. Available at http://dx.doi. 
org/10.21037/apm-21-1036

Data Sharing Statement: Available at http://dx.doi. org/10.21037/apm-21-1036

Conflicts of Interest: All authors have completed the ICMJE uniform disclosure form (available at http://dx.doi. org/10.21037/apm-21-1036). The authors have no conflicts of interest to declare.

Ethical Statement: The authors are accountable for all aspects of the work in ensuring that questions related to the accuracy or integrity of any part of the work are appropriately investigated and resolved. This study was approved by the Research Ethics Committee of Xingtai People's Hospital: (2019) 064. All procedures performed in this study involving human participants were in accordance with the Declaration of Helsinki (as revised in 2013). The informed consent was taken from all the patients.

Open Access Statement: This is an Open Access article distributed in accordance with the Creative Commons Attribution-NonCommercial-NoDerivs 4.0 International License (CC BY-NC-ND 4.0), which permits the noncommercial replication and distribution of the article with the strict proviso that no changes or edits are made and the original work is properly cited (including links to both the formal publication through the relevant DOI and the license). See: https://creativecommons.org/licenses/by-nc-nd/4.0/.

\section{References}

1. ACOG Practice Bulletin No. 190: Gestational Diabetes Mellitus. Obstet Gynecol 2018;131:e49-e64.

2. Homayouni A, Bagheri N, Mohammad-AlizadehCharandabi S, et al. Prevention of Gestational Diabetes Mellitus (GDM) and Probiotics: Mechanism of Action: A Review. Curr Diabetes Rev 2020;16:538-45.

3. Alejandro EU, Mamerto TP, Chung G, et al. Gestational Diabetes Mellitus: A Harbinger of the Vicious Cycle of Diabetes. Int J Mol Sci 2020;21:5003.

4. D'Anna R, Santamaria A, Alibrandi A, et al. MyoInositol for the Prevention of Gestational Diabetes Mellitus. A Brief Review. J Nutr Sci Vitaminol (Tokyo) 2019;65:S59-S61.

5. Plows JF, Reynolds CM, Vickers MH, et al. Nutritional Supplementation for the Prevention and/or Treatment of Gestational Diabetes Mellitus. Curr Diab Rep 2019;19:73.
6. Pan J, Pan Q, Chen Y, et al. Efficacy of probiotic supplement for gestational diabetes mellitus: a systematic review and meta-analysis. J Matern Fetal Neonatal Med 2019;32:317-23.

7. Kuo CH, Li HY. Diagnostic Strategies for Gestational Diabetes Mellitus: Review of Current Evidence. Curr Diab Rep 2019;19:155.

8. Rosik J, Szostak B, Machaj F, et al. The role of genetics and epigenetics in the pathogenesis of gestational diabetes mellitus. Ann Hum Genet 2020;84:114-24.

9. Hod M, Kapur A, McIntyre HD, et al. Evidence in support of the International Association of Diabetes in Pregnancy study groups' criteria for diagnosing gestational diabetes mellitus worldwide in 2019. Am J Obstet Gynecol 2019;221:109-16.

10. Juan J, Yang H. Prevalence, Prevention, and Lifestyle Intervention of Gestational Diabetes Mellitus in China. Int J Environ Res Public Health 2020;17:9517.

11. Li SY, Ouyang YQ, Qiao J, et al. Technology-supported lifestyle interventions to improve maternal-fetal outcomes in women with gestational diabetes mellitus: A metaanalysis. Midwifery 2020;85:102689.

12. Al Hashmi I, Nandy K, Seshan V. Non-Medical Strategies to Improve Pregnancy Outcomes of Women with Gestational Diabetes Mellitus: A literature review. Sultan Qaboos Univ Med J 2019;19:e4-e10.

13. Reyes-Muñoz E, Guardo FD, Ciebiera M, et al. Diet and Nutritional Interventions with the Special Role of MyoInositol in Gestational Diabetes Mellitus Management. An Evidence-Based Critical Appraisal. Curr Pharm Des 2019;25:2467-73.

14. K C K, Zhang H, Vaidya A. Increased Incidence in False Positive Diagnosis of Gestational Diabetes Mellitus with $75 \mathrm{gm}$ Oral Glucose Tolerance Test: A Clinical Study in Chinese Women. J Nepal Health Res Counc 2019;17:103-8.

15. Wang Y, Luo BR. The association of body composition with the risk of gestational diabetes mellitus in Chinese pregnant women: A case-control study. Medicine (Baltimore) 2019;98:e17576.

16. He Z, Xie H, Liang S, et al. Influence of different diagnostic criteria on gestational diabetes mellitus incidence and medical expenditures in China. J Diabetes Investig 2019;10:1347-57.

17. Zhou Q, Wen S, Liu M, et al. Association between Gene Polymorphisms of Vitamin D Receptor and Gestational Diabetes Mellitus: A Systematic Review and MetaAnalysis. Int J Environ Res Public Health 2020;18:205. 
18. Simmons D. The benefits of the use of the new International Association of Diabetes in Pregnancy Study Groups guidelines for gestational diabetes mellitus. Aust N Z J Obstet Gynaecol 2020;60:486-8.

19. Schoenaker DAJM, Callaway LK, Mishra GD. The Role of Childhood Adversity in the Development of Gestational Diabetes. Am J Prev Med 2019;57:302-10.

20. Zamani B, Milajerdi A, Tehrani H, et al. Association of a plant-based dietary pattern in relation to gestational diabetes mellitus. Nutr Diet 2019;76:589-96.

21. Sakurai K, Eguchi A, Watanabe M, et al. Exploration of predictive metabolic factors for gestational diabetes mellitus in Japanese women using metabolomic analysis. J Diabetes Investig 2019;10:513-20.

(English Language Editor: D. Fitzgerald)

Cite this article as: Ma H, Qiao Z, Li N, Zhao Y, Zhang S. The relationship between changes in vitamin $A$, vitamin $E$, and oxidative stress levels, and pregnancy outcomes in patients with gestational diabetes mellitus. Ann Palliat Med 2021;10(6):66306636. doi: 10.21037/apm-21-1036 